\title{
4. $\ddot{U} b e r$ die Lichtabsorption wasseriger Lösungen von Kupfer- und Nickelsalzen; von E. Müller.
} (Auszug aus der Berliner Inaugural-Dissertation.)

\section{Einleitung.}

§ 1. Trifft Licht von der Intensität $J$ auf ein absorbierendes Medium von der Schichtdicke $d$, so ist die Intensität $J^{\prime}$ des austretenden Lichtes gegeben durch die Gleichung

$$
J^{\prime}=J \cdot 10^{-e d},
$$

worin $\varepsilon$, der sogenannte Extinktionskoeffizient, eine für das absorbierende Medium charakteristische Konstante ist, die von der Wellenlänge abhängt.

Für die Lösung einer absorbierenden Substanz in einem farblosen Lösungsmittel ändert sich der Extinktionskoeffizient mit der Konzentration. Hat die Verdünnung einer solchen Lösung keinen anderen EinfluB, als daß die Zahl der in $1 \mathrm{ccm}$ Lösung enthaltenen absorbierenden Moleküle verkleinert wird, so ist es wahrscheinlich, daB $\varepsilon$ der Konzentration $c$ proportional ist, daB also der Extinktionskoeffizient für die Konzentration $c$

$$
\varepsilon_{c}=A \cdot c
$$

ist. Diese Gleichung ist von Beer ${ }^{1}$ ) zuerst aufgestellt worden und wird als das Beersche Absorptionsgesetz bezeichnet. Nach demselben ist $A$, der sogenannte molekulare Extinktionskoeffizient der gelösten Substanz, eine von der Konzentration unabhängige Konstante.

Das Beersche Absorptionsgesetz gilt nur in wenigen Fällen streng, im allgemeinen ändert sich $A$ mit der Konzentration, so dab

$$
\varepsilon_{c}=A_{c} \cdot c
$$

1) A. Beer, Pogg. Ann. 86. p. 78. 1852. 
zu setzen ist. Führen wir diesen Wert in Gleichung (1) ein, so erhalten wir

$$
J^{\prime}=J .10-A_{c} c d .
$$

Über die beobachteten Abweichungen rom Beerschen Gesetz ${ }^{1}$ ) konnte man sich keine Rechenschaft geben, so lange man annahm, daB bei der Verdünnung einer Lösung die gelösten Moleküle ungeändert bleiben. Doch trifft diese Annahme jedenfalls nicht zu für elektrolytische Lösungen, in welchen nach Arrhenius ein Teil der gelösten Moleküle in die elektrisch geladenen Ionen zerfallen; der Dissoziationsgrad, d. i. das Verbältnis der Anzahl der dissoziierten zur Gesamtzahl der gelösten Moleküle, nimmt mit der Verdünnung zu und wird für unendliche Verdünnung gleich 1. Dieser Grenzzustand wird im allgemeinen schon bei endlichen Verdünnungen erreicht.

Aus dieser Theorie zieht Knoblauch ${ }^{2}$ ) folgende Schlüsse über die Abhängigkeit der Lichtabsorption von der Konzentration bei elektrolytischen Lösungen.

1. Das Absorptionsspektrum der konzentrierten, wenig dissoziierten Lösung eines Körpers muB verschieden sein von demjenigen der sehr verdünnten, nahezu rollkommen dissoziierten, weil die Absorption in der ersteren hauptsächlich durch die nichtdissoziierten, in der zweiten durch die dissoziierten Moleküle hervorgerufen wird.

2. Das Absorptionsspektrum verschiedener Salze desselben gefärbten Metalles (bez. derselben gefärbten Säure) muß bei hinreichender Verdünnung, bei welcher der Grenzzustand der Dissoziation erreicht ist, das gleiche werden, vorausgesetzt, $\mathrm{da} B$ in den dissoziierten Lösungen der betreffenden Salze die Absorption durch das gleiche Ion ausgeübt wird.

Knoblauch unterzog die von ihm aus der Dissoziationstheorie gezogenen Folgerungen einer eingehenden Prüfung, fand dieselben jedoch nicht in allen Fällen bestätigt.

1) Eine genaue Angabe der einschlägigen Arbeiten findet sich in meiner Berliner Dissertation (1903), sowie eine solche der neueren Arbeiten bei P. Vaillant, Ann. de chim. et phys. (7) 28. p. 213. 1903, welcher lihnliche Untersuchungen wie die hier mitgeteilten gemacht hat. Doch gibt mir die Arbeit des Hrn. Vaillant keine Veranlassung, die meinige zurückzuziehen.

2) O. Knoblauch, Wied. Ann. 43. p. 776. 1891. 
Ostwald ${ }^{1)}$ zeigte, daB die Knoblauchschen Beobachtungen nicht frei von Versuchsfehlern sind und pruffte selbst die zweite Knoblauchsche Folgerung an einer groBen Reihe von Salzen, indem er die Absorptionsspektra der verdünnten Lösungen unter geeigneten Bedingungen photographierte; dabei zeigte sich die Knoblauchsche Theorie aufs rollkommenste bestätigt. Seitdem hat sich mit den Beziehungen zwischen Lichtabsorption und Dissoziation eine große Reihe von Physikern und Chemikern beschäftigt.

Dio zahlreichen, bisher über die Lichtabsorption in Lösungen angestellten Versuche sind jedoch fast ausschlieBlich qualitativ; insbesondere ist die Knoblauch-Ostwaldsche Theorie über die Lichtabsorption verdünnter Lösungen solcher Salze, die ein gemeinsames farbiges lon besitzen, deren anderes Ion aber farblos ist, noch nie quantitativ geprüft worden. $Z_{\text {weck }}$ der vorliegenden Arbeit ist es, den Einflub der Konzentration auf den molekularen Extinktionskoeffizienten wässeriger Kupfersalzlösungen für ein möglichst großes Wellenlängengebiet und für möglichst viele Konzentrationen messend $\mathrm{zu}$ verfolgen. Für die wässerigen Lösungen der Nickelsalze ist dieser EinfluB qualitativ untersucht worden.

\section{Apparate und Methoden.}

§ 2. Zu meinen Versuchen diente ein von Franz Schmidt \& Hänsch nach den Angaben von Martens hergestelltes Kolorimeter. Fig. 1 stellt einen vertikalen Schnitt durch die Hauptteile des Apparates dar.

Das Licht einer Nernstlampe wird von den beiden Spiegeln $S$ reflektiert und trifft durch zwei Öfnungen $n_{1}$ und $n_{2}$ in dem Metallschirm $s \boldsymbol{s}$ auf das Milchglas $\boldsymbol{m} \boldsymbol{m}$. Von den beiden beleuchteten Stellen des Milchglases gehen zwei Strahlenbündel 1 und 2 aus, welche nach dem Durchgang durch die Flüssigkeitsröhren $D$ und $C$ bez. $B$ und $A$ zur

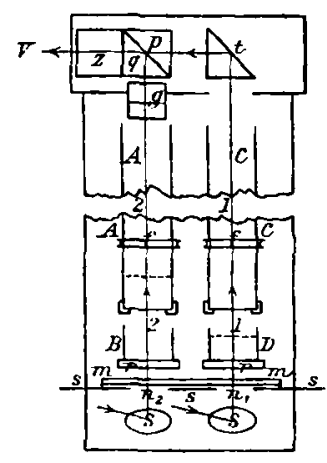

Fig. 1.

1) W. Ostwald, Zeitschr. f. phys. Chem. 9. p. 579. 1892. Annalen der Physik. IV. Folge. 12. 
photometrischen Vergleichsvorrichtung gelangen. Der wesentliche Teil der letzteren ist ein aus $z$ wei rechtwinkligen Prismen $p$ und $q$ bestehender Lummer-Brodhun-Würfel. Die Hypotenusenflächen der Prismen sind aneinander gekittet; ein Teil der Hypotenusenfläche von $q$ ist versilbert. Der versilberte Teil, ein zu den Prismenkanten senkrechter Streifen in der Mitte der Fläche, rellektiert das Bündel 2 in die Richtung $p v$; der unversilberte Teil läBt die von der versilberten Hypotenusenfläche des Prismas $t$ in die Richtung $t v$ reflektierten Strahlen 1 hindurchtreten.

Der weitere Verlauf der Strahlen wird am besten aus Fig. 2 klar, welche die Vergleichsvorrichtung von oben ge-

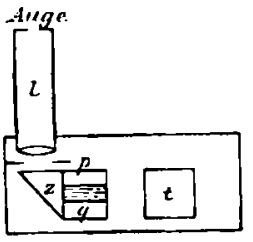

Fig. 2. sehen darstellt; die aus dem LummerBrodhun-Würfel $p q$ austretenden Strahlen werden durch das rechtwinklige Prisma $z$ in die Beobachtungslupe $l$ reflektiert.

Bevor das Bündel 2 in den LummerBrodhun-Wüfel eintritt, passiert es eine aus zwei Stücken zusammengekittete Glasplatte $g$ und durchläuft dabei dieselbe Glasdicke, wie das Bündel 1 im Prisma $t$. Dadurch ist erreicht, daB die Bündel 1 und 2 beim Durchgang durch die $\nabla$ ergleichsvorrichtung gleiche Bruchteile durch Reflexion und $\mathrm{Ab}$ sorption verlieren. Infolgedessen verschwinden bei richtiger Stellung der Nernstlampe und der Spiegel $s$ die Trennungs-

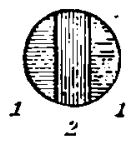
linien des Gesichtsfeldes vollständig. Dieses besteht aus drei Teilen; die Trennungslinien liegen vertikal (vgl. Fig. 3); der mittlere Teil wird rom Bündel 2, die beiden äuBeren Teile werden rom

Fig. 3. Bündel 1 beleuchtet.

Alles fremde Licht wurde durch ein schwarzes Tuch, das den ganzen Apparat umgab und auf den Metallschirm $s$ niederfiel, sorgfaltig ferngehalten.

Als Absorptionsgefäße dienten zwei kurze und zwei lange Glasröhren. Jede Röhre ist unten mit einem Schraubenverschlub versehen, welcher eine Spiegelglasplatte gegen das Rohrende drückt. Die Länge der kurzen Röhren betrug $4,35 \mathrm{~cm}$, die der langen 40,00 cm. Die Orientierung der Röhren im Apparat zeigt Fig. 1. Die kleinen GefäBe $B$ und $D$ warden 
in Ringfassungen $r$ eingesetzt, die großen $A$ und $C$ wurden durch aufgekittete Ringe $f f$ getragen, welche seitlich in passende Fassungen eingeschoben wurden.

Die Röhren wurden mit Quecksilber kalibriert und erwiesen sich als gut zylindrisch. Ferner war in die Röhren $A$ und $D$ in genau gleicher Höhe über dem Boden eine Marke eingeätzt (in Fig. 1 gestrichelt gezeichnet). Der Zweck dieser Marken wird aus dem Folgenden klar.

Bei den Versuchen waren die Röhren stets bis zum oberen Rande gefüllt und hier durch eine aufgeschobene Glasplatte verschlossen. Die ursprüngliche Absicht, mit freien Flüssigkeitsoberfächen $\mathrm{zu}$ arbeiten und etwaige Abweichungen vom Beerschen Gesetz durch Veränderung der Schichtdicke zu messen, muBte aufgegeben werden, da infolge der Brechung des Lichtes an den Flüssigkeitsmenisken die Beleuchtung der Gesichtsfelder ungleichmäBig wurde.

Für sehr stark absorbierende Substanzen standen anstatt der Röhren $B$ und $D$ andere AbsorptionsgefäBe zar Verfügung, welche derartig eingerichtet waren, daB mittels eines Schraubenverschlusses zwei Glasplatten gegen einen planparallel geschliffenen Glasring gepreBt wurden. Die durchstrablte Schichtdicke betrug hier nur $0,568 \mathrm{~cm}$.

Mit diesem Apparate wurde das Beersche Gesetz in der Weise geprüft, daB zunächst ohne absorbierende Medien auf Gleichheit der Gesichtsfelder eingestellt wurde. Darauf wurden auf beiden Seiten gleiche Mengen lichtabsorbierender Substanz, jedoch in verschiedenen Konzentrationen, und gleiche Mengen des Lösungsmittels in den Gang der Lichtstrahlen gebracht. Dies wurde dadurch erreicht, daB die Röhren $A$ und $D$ mit der zu untersuchenden Lösung bis zur Marke gefüllt wurden; darauf wurde in beiden Röhren bis obenhin das Lösungsmittel aufgegossen und die Gefäße mit Glasplatten verschlossen. Hierbei wurde selbstverständlich für gute Vermischung der Lösungen Sorge getragen. Dann werden offenbar auf beiden Seiten die gleichen Mengen absorbierender Substanz durchstrahlt, das Produkt aus molekularer Konzentration und Schichtdicke ist auf beiden Seiten das gleiche; unter der molekularen Konzentration ist die in 1 Liter der Lösung enthaltene Anzahl Gramm. moleküle des gelösten Salzes zu verstehen. 
Ferner wurden die beiden anderen Gefäße $B$ und $C$ mit dem Lösungsmittel gefüllt und oben mit Glasplatten verschlossen. Diese Anordnung bewirkt, daB auf beiden Seiten auch gleiche Mengen des Lösungsmittels durchstrahlt werden.

Bei dieser Versuchsanordnung nun müßten, die Richtigkeit des Beerschen Gesetzes vorausgesetzt, die Gesichtsfelder des Apparates gleiche Intensität und Farbe zeigen. Dies war jedoch bei den meisten untersuchten Lösungen nicht der Fall. $\mathrm{Um}$ die Abweichungen vom Beerschen Gesetz in den einzelnen Spektralregionen beurteilen zu können, bediente ich mich zunächst sogenannter Strahlenfilter. Solche Farbenfilter werden von den Schottschen Glaswerken in Jena hergestellt. Von

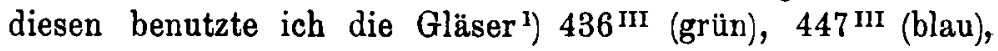
$452^{\text {III }}$ (violett), welche dem physikalischen Institut von der Firma Schott und Gen. überwiesen wurden. AuBerdem stand mir ein gewöhnliches rotes Kupferglas zur Verfügung, sowie zwei farbige Gläser, von denen das eine rot, gelb und grün, das andere grün, blau und violett durchlieB.

§ 3. Da, wie sich zeigte, die Abweichungen vom Beerschen Gesetz für Strahlen verschiedener Wellenlänge verschieden

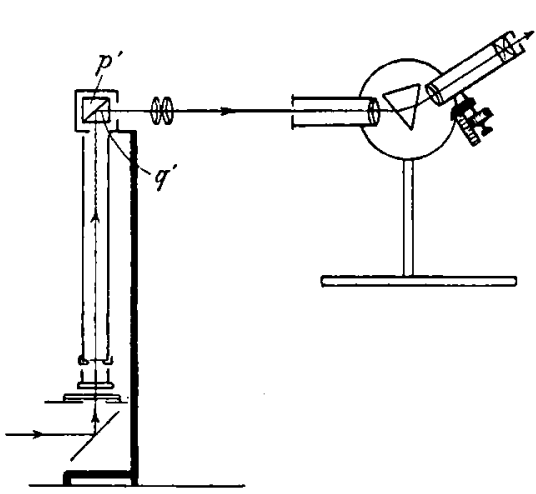

Fig. 4. groß sind, so muBten spektralphotometrische Messungen angestellt werden. $\mathrm{Zu}$ diesem $\mathrm{Z}$ weck wurde die Beobachtungslupe aus dem Kolorimeter entfernt. Das Prisma $z$ (Fig. 2) entwirft von dem LummerBrodhun-Würfel $p q$ ein virtuelles Bild $p^{\prime} q^{\prime}$ (Fig. 4). Dieses virtuelle Bild wurde mittels zweier achromatischer Objektive auf dem Kollimatorspalt eines Spektralapparates ${ }^{2}$ ) abgebildet. Das so entstehende reelle Bild besteht aus drei Teilen, von denen

1) R. Zsigm ondi, Zeitschr. f. Instrumentenk. 21. p. 97. 1901.

2) Die Firma Franz Schmidt \& Hansch hat mir das Spektroskop längere Zeit geliehen, wofür ich bestens danke. 
die beiden. äuBeren vom Strahlenbündel 1, das mittlere vom Bündel 2 beleuchtet werden.

Da diese drei Teile in vertikalen Trennungslinien zusammenstoBen, so wurde das Spektroskop so orientiert, daB sein Spalt horizontale Lage hatte, also das Beobachtungsfernrohr in einer Vertikalebene drehbar war (Fig. 4). Man erblickt in demselben drei nebeneinander liegende Spektren, von denen die beiden äußeren das Absorptionsspektrum der konzentrierten, das mittlere das der verdünnten Lösung darstellen. In der Okularblende des Fernrohres wurde ein horizontaler Spalt angebracht, durch den aus allen drei Spektren ein schmaler Bezirk ausgeblendet wurde. Die Bewegung des Fernrohres erfolgte mittels einer Mikrometerschraube, deren MeBtrommel mit Hilfo einiger bekannter Spektrallinien auf Wellenlängen geeicht wurde. Vor jeder Messung wurde die richtige photometrische Einstellung des ganzen Apparates kontrolliert.

Ist $J$ die Intensität der beiden auffallenden Lichtbündel 1 und 2, bezeichnet man ferner Intensität des austretenden Lichtes, molekularen Extinktionskoeffizienten, molekulare Konzentration und Schichtdicke mit $J_{1}^{\prime}, A_{1}, c_{1}, d_{1}$ für die konzentrierte, mit $J_{2}^{\prime}, A_{2}, c_{2}, d_{2}$ für die verdünnte Lösung, so gilt infolge der Gleichung (4):

$$
\begin{aligned}
& J_{1}^{\prime}=J .10^{-A_{1} c_{1} d_{1}}, \\
& J_{2}^{\prime}=J .10^{-A_{2} c_{2} d_{2}} .
\end{aligned}
$$

Hieraus folgt, wenn man berücksichtigt, daB infolge der Versuchsanordnung $c_{1} d_{1}=c_{2} d_{2}$ ist:

$$
A_{1}-A_{2}=\frac{1}{c_{1} d_{1}} \log \frac{J_{2}^{\prime}}{J_{1}^{\prime}} \text {. }
$$

Die Messung des Verhältnisses $J_{2}^{\prime} / J_{1}^{\prime}$ geschah durch photographisch geschwärzte Platten. Es wurden einige Platten verschieden lange belichtet, und es ergab sich dann bei der Entwickelung ein verschiedener Grad der Schwärzung. ${ }^{1}$ ) Diese Platten wurden unmittelbar auf die Milchglasplatte $m$ gelegt, und zwar auf dasjenige der Felder $n$, dessen zugehöriges Gesichtsfeld das hellere war. Darauf wurde das Beobachtungs-

1) Für die Anfertigung der Platten bin ich Hrn. Dr. R. Defregger zu Dank verpflichtet. 
fernrohr auf diejenige Wellenlänge $\lambda$ eingestellt, für die dann Gleichheit der Felder vorhanden war.

Sei $J_{2}^{\prime}>J_{1}^{\prime}$ - dies war bei den Substanzen, an welchen Messungen angestellt wurden, der Fall - und schwäche die betreffende Platte das auffallende Licht um $p$ Proz., so haben wir die Gleichung:

$$
J_{2}^{\prime}-\frac{J_{8}^{\prime} \cdot p}{100}-J_{1}^{\prime} .
$$

Führt man dies in Gleichung (5) ein, so geht dieselbe über in:

$$
A_{1}-A_{2}=\frac{1}{c_{1} d_{1}} \log \cdot \frac{100}{100-p} .
$$

Hat man für eine bestimmte Anfangskonzentration $c_{1}$ die Differenz $A_{1}-A_{2}$ gemessen, so findet man, indem man die Lösung im GefäB $D$ durch Wasser ersetzt $\left(A_{1}=0\right)$, aus dieser Gleichung $A_{2}$, dadurch kennt man dann auch die $A_{1}$.

Die GröBe $p$ ist für photographisch geschwärzte Platten, wie sich durch Messung der Absorption einer Platte für rerschiedene Wellenlängen mit dem Königschen Spektralphotometer ergab, von der Farbe des Lichtes so gut wie unabhängig. Daher konnte $p$ mit dem Martensschen Polarisationsphotometer für weißes Licht ${ }^{1}$ ) gemessen werden.

Die Größe $p$ hatte für die benutzen Platten folgende Werte:

Tabelle 1 .

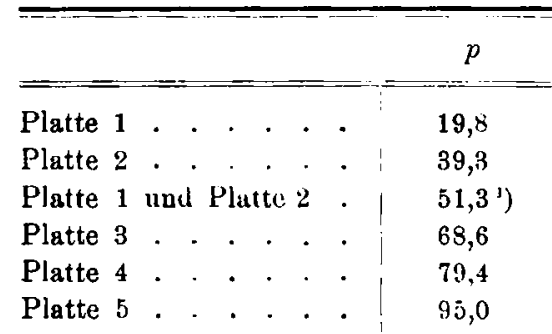

III. Beobachtungsresultate.

Bevor wir zur Darstellung der Beobachtungsresultate übergehen, sei folgendes bemerkt: Wie bereits auf p. 772 erwähnt, ändert sich im allgemeinen der molekulare Extinktionskoeffizient

1) F. F. Martens, Phys. Zeitschr. 1. p. 299.1900. 
mit der Konzentration; es zeigte sich jedoch, daB derselbe bei zunehmender Verdünnung einen Grenzwert erreicht; wir bezeichnen denselben mit $\boldsymbol{A}_{0}$. Wurde nun die Konzentration $c_{1}$ in Röhre $D$ (Fig. 1) so groB wie möglich gewählt, so zeigte sich die Verdünnung in Röhre $A$ bei allen untersuchten Substanzen mit Ausnahme von Kupferacetat schon so weit vorgeschritten, daB $A$ bereits seinen Grenzwert erreicht hatte. Man kann daher für diese Substanzen in Gleichung (6) durch $A_{0}$ ersetzen.

Unter Berücksichtigung der Knoblauch-Ostwaldschen Theorie mögen nun im folgenden die Beobachtungsresultate zusammengestellt werden.

§ 4. Kupfersulfat: $\mathrm{CuSO}_{4}+5 \mathrm{H}_{2} \mathrm{O}$. Das Beersche Gesetz zeigte sich für alle durchgelassenen Farben bestätigt. In Tab. 2 sind die vom Verfasser bestimmten molekularen Extinktionskoeffizienten zusammengestellt.

Tabelle 2.

$\mathrm{CuSO}_{4}$. Bezeichnungen vgl. p. 773 und 775.

\begin{tabular}{|c|c|c|}
\hline \multirow{2}{*}{$p$} & \multicolumn{2}{|c|}{$c_{2}=0,091$} \\
\hline & $\lambda$ in $\mu \mu$ & $A$ \\
\hline 19,8 & - & - \\
\hline 39,3 & 512 & 0,060 \\
\hline 51,3 & 524 & 0,088 \\
\hline 68,6 & 538 & 0,139 \\
\hline 79,4 & 549 & 0,189 \\
\hline 95,0 & 569 & 0,358 \\
\hline
\end{tabular}

Von F. Grünbaumn ${ }^{1}$ ) sind neuerdings mit dem KönigMartensschen Spektralphotometer Absorptionsmessungen an wässerigen $\mathrm{CuSO}_{4}$-Lösungen angestellt worden, die als die genauesten der über dies Salz veröffentlichten Messungen gelten dürfen. In Tab. 3 ist ein Teil der aus den Grünbaumschen und Ewanschen ${ }^{2}$ ) Messungen sich ergebenden molekularen Extinktionskoeffizienten $A$ zusammengestellt.

1) F. Grünbaum, lnaug.Diss. Berlin 1902.

2) T. Ewan, Phil. Mag. (5) 33. p. 317. 1892. 
Tabelle 3 .

$\mathrm{CuSO}_{4}$.

\begin{tabular}{c|c||c|c}
\hline \multicolumn{2}{c||}{ Ewan } & \multicolumn{2}{c}{ Grünbaum } \\
\hline$\lambda$ in $\mu \mu$ & $A$ & $\lambda$ in $\mu \mu$ & $A$ \\
\hline \hline 495 & 0,024 & 480 & 0,010 \\
510 & 0,050 & 492 & 0,017 \\
526 & 0,090 & 500 & 0,023 \\
543 & 0,164 & 508 & 0,036 \\
563 & 0,298 & 521 & 0,063 \\
& & 534 & 0,106 \\
& & 546 & 0,167 \\
& & 560 & 0,273 \\
& & 568 & 0,357
\end{tabular}

Wie man ans Fig. 5 sieht, stimmen die Werte des Verfassers mit denen von Grünbaum nur für große Wellenlängen

Kupfersulfat.

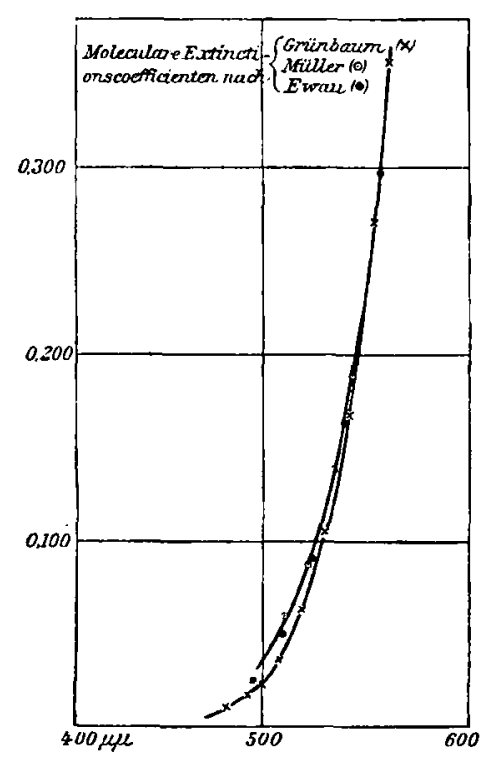

Fig. 5. gut überein. Dies scheint daher zu rühren, daB Verfasser eine weiBe Lichtquelle (Nernstlampe) benutzte, während Hr. Grünbaum sich monochromatischen Lichtes bediente.

Vergleicht man nämlich mit den Werten des Verfassers diejenigen ron Ewan, der sich ebenfalls einer weißen Lichtquelle bediente, so sieht man, daB diese Werte gut übereinstimmen (vgl. Fig. 5).

Die Absorption des roten Spektralendes ist, wie wir sehen werden, bei allen Kuprisalzen vorhanden; sie wird also durch das allen gemeinsame Kupfer bewirkt sein.

Daraus, daB in allen anderen Farben des Spektrums keine Absorption stattfindet, und daB das Beersche Gesetz im sichtbaren Gebiet erfüllt ist, folgt erstens, daB das Ion $\mathrm{SO}_{4}$ 
farblos ist, zweitens, daB das Cu seine spezifische Absorption im Rot beibehält, wenn es mit $\mathrm{SO}_{4}$ zum Molekül $\mathrm{CuSO}_{4}$ zusammentritt.

Unaufgeklärt bleibt die Tatsache, daß völlig wasserfreies $\mathrm{CuSO}_{4}$ farblos, $\mathrm{CuSO}_{4}+1 \mathrm{H}_{2} \mathrm{O}$ grünlich ist.

Von der Temperatur zeigt sich die Lichtabsorption in wässeriger $\mathrm{CuSO}_{4}$-Lösung unabhängig.

§ 5. Kupferchlorid: $\mathrm{CuCl}_{2}+2 \mathrm{H}_{2} \mathrm{O}$. In Tab. 4 sind die gemessenen Werte $A_{1}-A_{0}$ bez. $A_{0}$ zusammengestellt.

Tabelle 4.

$\mathrm{CuCl}_{2}$. Bezeichnungen vgl. p. 773 und 775 .

\begin{tabular}{|c|c|c|c|c|c|c|c|c|c|c|}
\hline \multirow[b]{2}{*}{$p$} & \multicolumn{2}{|c|}{$c_{1}=2,668$} & \multicolumn{2}{|c|}{$c_{1}=2,002$} & \multicolumn{2}{|c|}{$c_{1}=1,336$} & \multicolumn{2}{|c|}{$c_{1}=0,667$} & \multicolumn{2}{|c|}{$c_{2}=0,073$} \\
\hline & $\begin{array}{l}z \\
z \\
\underline{z} \\
z\end{array}$ & 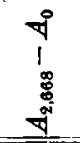 & $\begin{array}{l}z \\
\Xi \\
z \\
\alpha\end{array}$ & 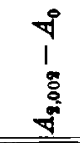 & 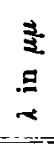 & 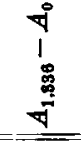 & $\begin{array}{l}\frac{3}{3} \\
. \Xi \\
z\end{array}$ & $\begin{array}{l}0 \\
1 \\
\vdots \\
8\end{array}$ & $\begin{array}{l}\Xi \\
\Xi \\
\Xi\end{array}$ & $A_{0}$ \\
\hline 19 & & & 515 & & & & & & 19 & \\
\hline 39 & & & 498 & & & 0,0 & 428 & 0,075 & & \\
\hline 51,3 & 508 & & 49 & & 468 & 0,05 & - & - & 2 & \\
\hline 68 & 50 & & 48 & & & & - & - & & \\
\hline & 800 & & 483 & 0,079 & 457 & 0,119 & - & - & 554 & 0,237 \\
\hline 95 & & & & & - & - & - & - & - & - \\
\hline
\end{tabular}

Der Verlauf der Kurven (Fig. 6) gibt ein Bild von den Abweichungen vom Beerschen Gesetz. Würde dieses erfullt sein, so müBte für alle Wellenlängen $A_{1}-A_{0}=0$ sein; die Kurven müBten mit der Abszissenachse zusammenfallen. Am einen Spektralende schmiegen sich die Kurven in der Tat an die Abszissenachse an; da ist das Gesetz erfüllt. Am anderen Ende steigen sie steil empor. Der Ort dieses Anstieges rückt mit zunehmender Verdünnung nach kleinen Wellenlängen. Je mehr also die Verdünnung zunimmt, für um so mehr Wellenlängen erreicht $A$ seinen Grenzwert $A_{0}$. Wird die Anfangsverdünnung $\left(c_{1}\right)$ so gro $B$, da $B$ die Kurve $A_{1}-A_{0}$ im ganzen sichtbaren Gebiet mit der Abszissenachse zusammenfällt, so hat $A$ innerhalb dieses Gebietes seinen Grenzwert erreicht. 
Dieser Grenzwert ist, wie man sich überzeugt (vgl. Fig. 10), in Übereinstimmung mit der Knoblauch-Ostwaldschen Theorie für Kupferchlorid der gleiche wie für Kupfersulfat.

Aus dem Gang der Differenzkurven mit zunehmender Verdünnung kann man mit groBer Wahrscheinlichkeit folgern,

Kupferchlorid.

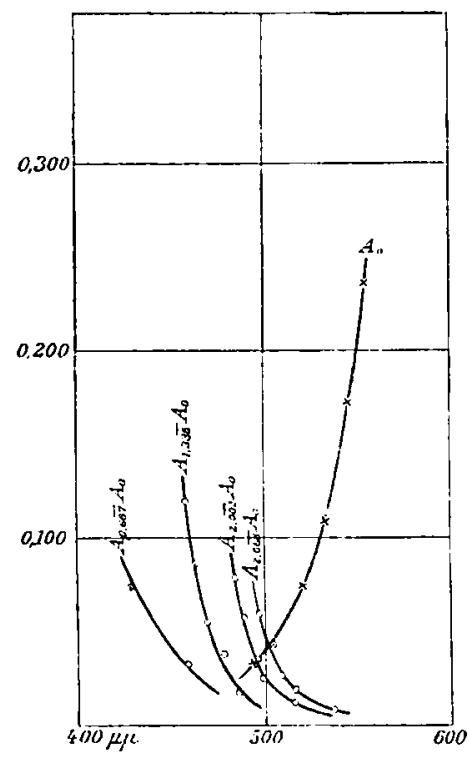

Fig. 6. daB im Ultraviolett noch merkliche Abweichungen vom Beerschen Gesetz bestehen, wenn dasselbe im sichtbaren Gebiet schon erfüllt ist. Man muB also sehr vorsichtig sein, wenn man nur die Farbe einer Lösung beobachtet hat und hieraus Schlüsse auf den Dissoziationsgrad ziehen will; wenn, wie beim $\mathrm{CuSO}_{4}$, das Beersche Gesetz im ganzen sichtbaren Gebiet erfüllt ist, so können immerhin Abweichungen von demselben noch im Ultraviolett oder Ultrarot vorhanden sein.

Die Lichtabsorption der wässerigen $\mathrm{CuCl}_{2}$-Lösungen hängt nicht nur von der Konzentration, sondern auch von der Temperatur in hohem Grade ab; und zwar hat Erhöhung der Temperatur auf die Lichtabsorption einer $\mathrm{CuCl}_{2}$-Lösung denselben EinfluB wie Vergrößerung, Erniedrigung der Temperatur denselben EinfluB wie Verringerung der Konzentration. So wird z. B. die verdünnte (blave) Lösung beim Erwärmen grün, die konzentrierte (grüne) Lösung beim Abkühlen blau; mit der früheren Temperatur kehrt auch die ursprüngliche Farbe zurück.

Wasserfreies $\mathrm{CuCl}_{2}$ ist ein braungelbes Pulver. ${ }^{1}$ )

§6. Kupferbromid: $\mathrm{CuBr}_{2}$. Tab.5 enthält die gemessenen Werte $A_{1}-A_{0}$ bez. $A_{0}$.

1) U. Dammer, Handb. d. anorg. Chem. (II) 2. p. 695. 1894. 
Lichtabsorption wässeriger Lösungen etc.

Tabelle 5.

$\mathrm{CuBr}_{2}$. Bezeichnungen vgl. p. 773 und 775.

\begin{tabular}{|c|c|c|c|c|c|c|c|c|c|c|}
\hline \multirow[b]{2}{*}{$p$} & \multicolumn{2}{|c|}{$c_{\mathrm{i}}=1,390$} & \multicolumn{2}{|c|}{$c_{1}=0,737$} & \multicolumn{2}{|c|}{$c_{1}=0,545$} & \multicolumn{2}{|c|}{$c_{1}=0,369^{\circ}$} & \multicolumn{2}{|c|}{$c_{2}=0,059$} \\
\hline & $\begin{array}{l}3 \\
\equiv \\
\approx\end{array}$ & $\begin{array}{c}0 \\
1 \\
0 \\
0 \\
0\end{array}$ & $\begin{array}{l}\vec{z} \\
\underline{\Sigma} \\
\underline{z}\end{array}$ & 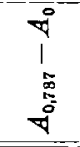 & 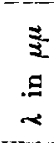 & $\begin{array}{l}\nabla^{\circ} \\
1 \\
10 \\
0 \\
0 \\
0\end{array}$ & $\begin{array}{l}z \\
\Xi \\
\Xi \\
z\end{array}$ & $\begin{array}{l}70 \\
1 \\
8 \\
80\end{array}$ & $\begin{array}{l}z \\
z \\
a \\
\alpha\end{array}$ & $A_{0}$ \\
\hline 19,8 & - & - & 584 & 0,030 & 555 & 0,040 & 486 & 0,060 & 499 & 0,040 \\
\hline 39,3 & 629 & 0,275 & 572 & 0,068 & 520 & 0,091 & 457 & 0,135 & 523 & 0,091 \\
\hline 51,3 & 620 & 0,401 & 562 & 0,099 & 505 & 0,134 & 448 & 0,198 & 535 & 0,134 \\
\hline 68,6 & 609 & 0,636 & 541 & 0,157 & 488 & 0,212 & - & - & 549 & 0,212 \\
\hline 79,4 & 586 & 0,870 & 523 & 0,214 & 475 & 0,290 & - & - & 559 & 0,290 \\
\hline 95,0 & 542 & 1,647 & 493 & 0,406 & - & - & - & - & - & - \\
\hline
\end{tabular}

Die Kurven (Fig. 7) rerlaufen ebenso wie bei $\mathrm{CuCl}_{2}$, insbesondere ergibt sich in Übereinstimmung mit der Knob-

Kupferbromid.

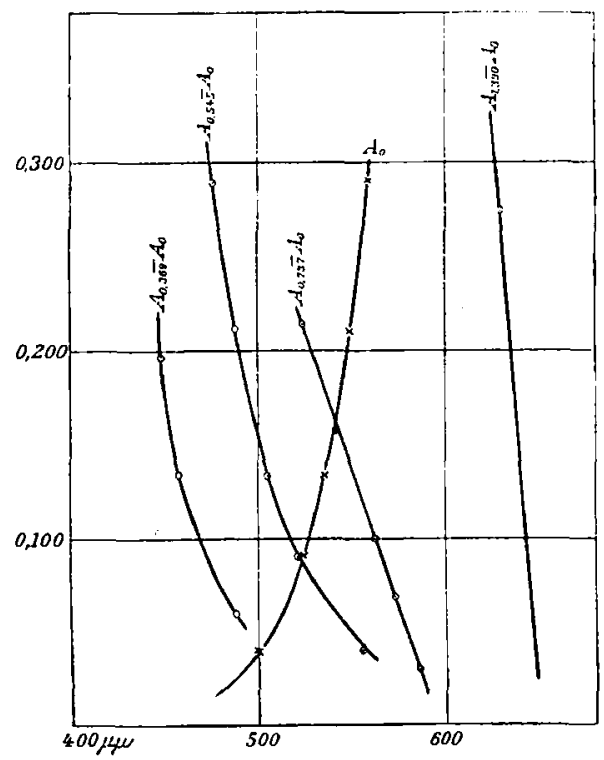

Fig. 7.
Kupfernitrat.

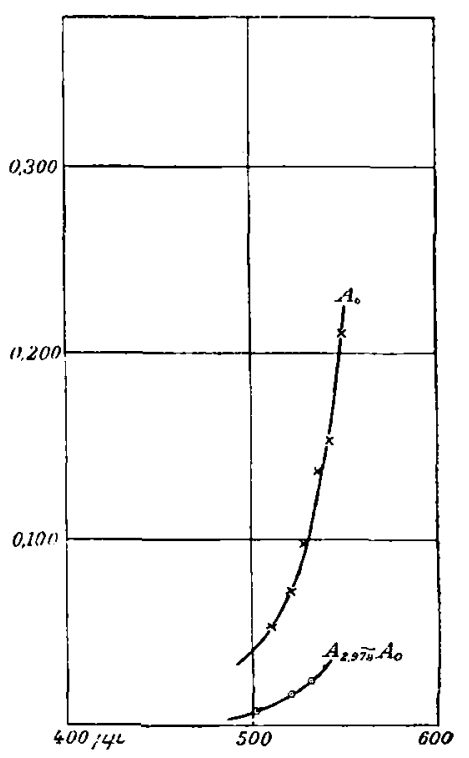

Fig. 8.

lauch-Ostwaldschen Theorie für $A_{0}$ dieselbe Kurve wie bei $\mathrm{CuSO}_{4}$ und $\mathrm{CuCl}_{2}$ (vgl. Fig. 10). Man ersieht jedoch aus den 
Kurven, daB die Abweichungen rom Beerschen Gesetz hier gröBer sind als bei $\mathrm{CuCl}_{2}$. Auch für wässerige Lösungen von $\mathrm{CuBr}_{2}$ gilt der Satz: Erhöhung der Temperatur hat auf die Lichtabsorption denselben EinfluB wie VergröBerung, Erniedrigung der Temperatur denselben Einfluß wie Verringerung der Konzentration. Eine analoge Erscheinung ist vom flüssigen Brom bekannt ${ }^{1}$ ); kühlt man dasselbe auf $-180^{\circ} \mathrm{ab}$, so geht die dunkle rotbraune Farbe in ein helles Gelbrot über.

§ 7. Kupfernitrat: $\mathrm{Cu}\left(\mathrm{NO}_{3}\right)_{2}+6 \mathrm{H}_{2} \mathrm{O}$. Hier ist das Beersche Gesetz am blauen Spektralende erfullt, im Rot dagegen absorbiert die konzentrierte Lösung stärker als die verdünnte.

In Tab. 6 sind die gemessenen Werte $A_{1}-A_{0}$ bez. $A_{0}$ zusammengestellt.

Tabolle 6.

$\mathrm{Cu}\left(\mathrm{NO}_{3}\right)_{\text {. }}$. Bezeichnungen $\nabla g l$. p. 773 und 775 .

\begin{tabular}{c||c|c|c|c}
\hline \multicolumn{1}{c||}{} & \multicolumn{2}{c|}{$c_{1}=2,979$} & \multicolumn{2}{c}{$c_{2}=0,081$} \\
\cline { 2 - 5 } & $\lambda$ in $\mu \mu$ & $A_{2,979}-A_{0}$ & $\lambda$ in $\mu \mu$ & $A_{0}$ \\
\hline 19,8 & 501 & 0,007 & 510 & 0,053 \\
39,3 & 521 & 0,017 & 521 & 0,072 \\
51,3 & 532 & 0,024 & 529 & 0,098 \\
68,6 & - & - & 537 & 0,137 \\
79,4 & - & - & 542 & 0,155 \\
95,0 & - & - & 550 & 0,212
\end{tabular}

Auch hier besitzt $A_{0}$ in Übereinstimmung mit der Knoblauch-Ostwaldschen Theorie dieselben Werte (Fig. 10), wie bei den bisher untersuchten Kupfersalzen.

Erwärmt man das wasserhaltige Salz, so schmilzt dasselbe in seinem Kristallwasser; dampft man diese Lösung ein, so wird sie grün.

8 8. Kupferacetat: $\mathrm{Cu}\left(\mathrm{C}_{2} \mathrm{H}_{9} \mathrm{O}_{2}\right)_{2}+\mathrm{H}_{2} \mathrm{O}$. Das Beersche Gesetz ist, wie beim Nitrat, am blauen Spektralende erfüllt, am roten absorbiert die konzentrierte Lösung stärker als die verdünte.

1) F. F. Martens, Verhandl. d. Deutschen physik. Gesellsch. 4. p. 141. 1902 . 
Tabelle 7.

$\mathrm{Cu}\left(\mathrm{C}_{8} \mathrm{H}_{3} \mathrm{O}_{2}\right)_{2}$. Bezeichnungen vgl. p. 773.

\begin{tabular}{|c|c|c|c|c|c|c|c|c|c|c|c|c|}
\hline \multirow[b]{2}{*}{$p$} & \multicolumn{2}{|c|}{$c_{1}=0,111$} & \multicolumn{2}{|c|}{$c_{1}=0,083$} & \multicolumn{2}{|c|}{$c_{1}=0,056$} & \multicolumn{2}{|c|}{$c_{2}=0,012$} & \multicolumn{2}{|c|}{$c_{2}=0,009$} & \multicolumn{2}{|c|}{$r_{2}=0,006$} \\
\hline & $\begin{array}{l}\vec{z} \\
\Xi \\
\approx\end{array}$ & एँ & $\begin{array}{l}\frac{j}{\Sigma} \\
\Xi \\
\Sigma \\
\end{array}$ & 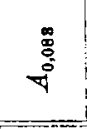 & $\begin{array}{l}\overrightarrow{3} \\
\Xi \\
\approx\end{array}$ & : & $\begin{array}{l}3 \\
\Xi \\
\approx \\
\sim\end{array}$ & $\stackrel{0}{\square}$ & $\begin{array}{l}3 \\
E \\
E\end{array}$ & $\begin{array}{l}\text { के } \\
\text { क्षें } \\
\text { च }\end{array}$ & $\begin{array}{l}\underline{z} \\
\Xi \\
\Xi \\
2\end{array}$ & ${ }_{0}^{\circ}$ \\
\hline$\theta, 8$ & 523 & 368 & 523 & & 535 & & 525 & & 538 & 0,264 & 90 & 800 \\
\hline 9,3 & 543 & 0,908 & 552 & 1,138 & 571 & & 543 & 0,4 & 55 & 0,598 & 569 & 897 \\
\hline 1,3 & 555 & 1,365 & 566 & 1,773 & 583 & 2,640 & 552 & & 566 & 0,873 & & 310 \\
\hline 68,6 & 571 & 2,268 & 584 & 2,765 & 610 & 4,867 & 566 & 1,038 & 579 & 1,385 & 597 & 2,077 \\
\hline 79,4 & 585 & 3,27 & 600 & 4,353 & - & - & 576 & 1,420 & 592 & 1,893 & 612 & 2,839 \\
\hline 5,0 & - & & - & - & - & - & - & $\pi$ & 612 & 3,585 & -1 & - \\
\hline
\end{tabular}

Tab. 7 enthält die molekularen Extinktionskoeftizienten $\left.{ }^{1}\right)^{2}$ ) für verschiedene Konzentrationen.

Für Kupferacetat konnte $A_{0}$ nicht gemessen werden, da bei diesem Salz $A$ erst bei einer Verdünnung konstant zu werden scheint, bei der wegen der schwachen Färbung Absorptionsmessungen nicht mehr angestellt werden konnten. Man sieht aber aus Fig. 9, wie auch hier die molekularen Extinktionskurven sich der Kurve $A_{0}$ der anderen Kupfersalze (dieselbe ist gestrichelt gezeichnet) mit wachsender Verdünnung nähern.

Über die Abhängigkeit der Lichtabsorption von der Temperatur wurden bei diesem Salz keine Versuche angestellt, da sich dasselbe schon bei geringer Erwärmung zersetzt.

§ 9. Kupferchlorat: $\mathrm{Cu}\left(\mathrm{ClO}_{3}\right)_{2}+6 \mathrm{H}_{2} \mathrm{O}$. Dieses Salz wurde nur qualitativ untersucht. Das Beersche Gesetz zeigt sich für fast alle durchgelassenen Farben erfüllt, nur am alleräußersten roten Ende zeigen sich geringe Spuren von Abweichungen, indem die konzentrierte Lösung etwas stärker absorbiert als die verdünnte. und 775).

1) Hier muAten $A_{1}$ und $A_{2}$ einzeln bestimmt werden (vgl. p. 774

2) Kupferacetat ist in Wasser viel weniger löslich als die anderen untersuchten Salze; da es trotzdem stark färbt, so sind die molekularen Extinktionskoeffizienten hier von etwa $10 \mathrm{mal}$ höherer Größenordnung als bei jenen. Der MaBstab der Ordinaten in Fig. 9 ist deswegen $10 \mathrm{mal}$ kleiner gewăhlt als für die anderen Kurven. 
Erwärmt man die blauen, wasserhaltigen Kristalle, so schmelzen dieselben im Kristallwasser; bei weiterem Eindampfen wird diese Lösung grün.

$\S 10$. Es wurde bereits auf p. 778 darauf hingewiesen, daB bei $\mathrm{CuCl}_{2}$ (und ebenso bei $\mathrm{CuBr}_{2}$ ) Abweichungen vom Beerschen Gesetz wahrscheinlich auch im Ultraviolett vorhanden sind. In der Tat ergaben Versuche, welche $\mathrm{Hr}$.

Kupferacetat.

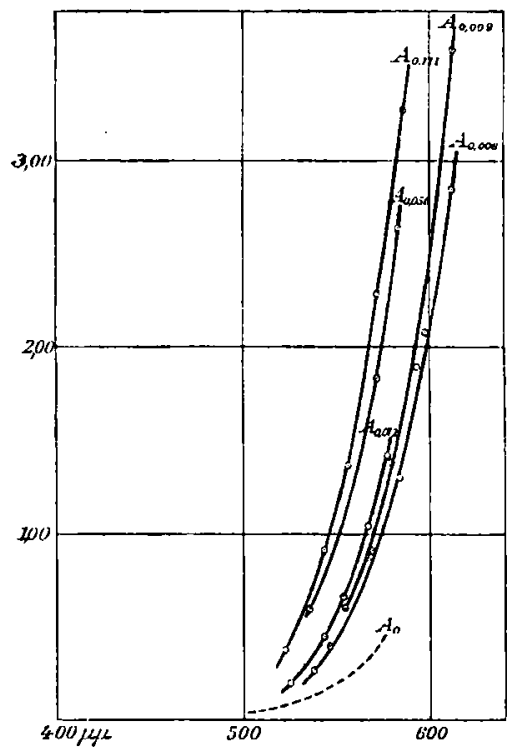

Fig. 9.

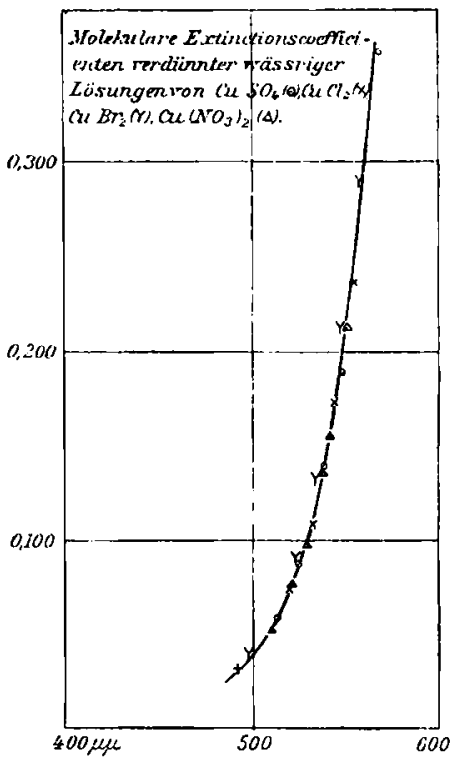

Fig. 10.

Martens über das optische Verhalten von $\mathrm{CuCl}_{2}$-Lösungen im Ultraviolett angestellt hat, folgendes:

Die Brechungsexponenten der konzentrierten Lösung fangen bei etwa $310 \mu \mu$ an, nach der Seite der kleinen Wellenlängen hin erheblicher anzusteigen, von $298 \mu \mu$ an ist die Lösung auch bei einer Schichtdicke von nur wenigen hundertstel Millimetern undurchlässig; dies Verhalten erklärt sich, wenn man annimmt, daB reines Chlor seine Eigenschwingung bei $210 \mu \mu$

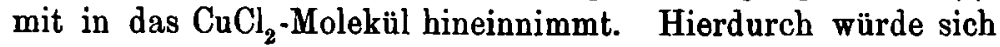
auch die Absorption blauer Strablen erklären, die ja auch flüssigem Chlor eigentümlich ist. Bei verdünnten $\mathrm{CuCl}_{2}$ - 
Lösungen steigen die Brechungsexponenten erst bei kleineren Wellenlängen an.

Bei der konzentrierten wässerigen $\mathrm{CuBr}_{2}$-Lösung fällt die Eigenschwingung im Ultraviolett, wie Hr. Martens fand ${ }^{1}$, etwa mit der von $\mathrm{Br}_{2}$ zusammen; die letztere liegt, entsprechend dem höheren Atomgewicht von $\mathrm{Br}^{2}$ ), bei größeren Wellenlängen als die von $\mathrm{Cl}_{2}$, nämlich bei $310 \mu \mu$. Hieraus dürfte es sich erklären, daB die Abweichungen vom Beerschen Gesetz bei $\mathrm{CuBr}_{2}$ gröBer sind als bei $\mathrm{CuCl}_{2}$. Es scheint demnach, daB Brom seine Absorption mit in das $\mathrm{CuBr}_{2}-\mathrm{Molekül}$ hineinnimmt. In der verdünnten Lösung macht sich der stärkere Anstieg des Brechungsexponenten und der Absorption erst bei viel kleineren Wellenlängen bemerkbar, nämlich zwischen 220 und $270 \mu \mu$.

Auch die Farblosigkeit des $\mathrm{SO}_{4}$ steht in Übereinstimmung mit Versuchen des Hrn. Martens ${ }^{3}$, der wahrscheinlich gemacht hat, dab die Eigenschwingung des $\mathrm{SO}_{4}$ unterhalb $100 \mu \mu$ liegt, also keinen Einflub im sichtbaren Gebiet ausüben wird.

§ 11. Nickelsalze. $\nabla$ on den Salzen des Nickeis wurden dieselben untersucht, wie vom Kupfer, die meisten jedoch nur qualitativ. Das Beersche Gesetz zeigte sich für alle durchgelassenen Farben erfüllt beim Sulfat und Nitrat. Bei den anderen Salzen sind die Abweichungen von derselben Art wie bei den entsprechenden Kupfersalzen, doch sind sie ihrer GröBe nach bei weitem geringer als bei diesen. Immerhin sind sie noch recht beträchtlich beim Bromür $\left(\mathrm{NiBr}_{2}\right)$ und Chlorür $\left(\mathrm{NiCl}_{2}\right)$; dagegen sind beim Acetat $\left(\mathrm{Ni}\left(\mathrm{C}_{2} \mathrm{H}_{3} \mathrm{O}_{2}\right)_{2}\right)$ und Chlorat $\left(\mathrm{Ni}\left(\mathrm{ClO}_{3}\right)_{2}\right)$ nur ganz geringe Abweichungen vom Beerschen Gesetz nachweisbar.

Ähnlich wie die Kupfersalze zeigen auch alle Nickelsalze in verdünnter Lösung gleiche Farbe: $\mathrm{NiBr}_{2}$ ist braun, $\mathrm{NiCl}_{2}$ gelbgrün, $\mathrm{NiSO}_{4}$ und $\mathrm{Ni}\left(\mathrm{NO}_{3}\right)_{2}$ sind grasgrün in konzentrierter Lösung; in verdünnter Lösung zeigen diese Salze den gleichen grünen Farbenton, und man darf wohl annehmen, dab auch hier sich für alle Salze dieselbe Kurve $A_{0}$ ergibt. Die molekularen Extinktionskoeffizienten sind für Nickelnitrat gemessen worden und in Tab. 8 zusammengestellt.

1) 2) 3) F. F. Martens, 1. c. 
Tabelle 8. $\mathrm{Ni}\left(\mathrm{NO}_{\mathbf{3}}\right)_{\mathbf{2}}$.

\begin{tabular}{|c|c|c|}
\hline \multirow{2}{*}{$p$} & \multicolumn{2}{|c|}{$c_{9}=0,053$} \\
\hline & $\lambda$ & $A$ \\
\hline 19,8 & $\begin{array}{l}511 \\
489\end{array}$ & 0,045 \\
\hline 39,3 & $\begin{array}{l}534 \\
480\end{array}$ & 0,102 \\
\hline 51,3 & $\begin{array}{l}553 \\
474\end{array}$ & 0,149 \\
\hline 68,6 & $\begin{array}{l}565 \\
467\end{array}$ & 0,236 \\
\hline 79,4 & $\begin{array}{l}577 \\
457\end{array}$ & 0,322 \\
\hline
\end{tabular}

Wie man aus Fig. 11 sieht, absorbiert $\mathrm{Ni}$ sowohl das rote, als auch das blaue Ende des sichtbaren Spektrums.

Nickelnitrat.

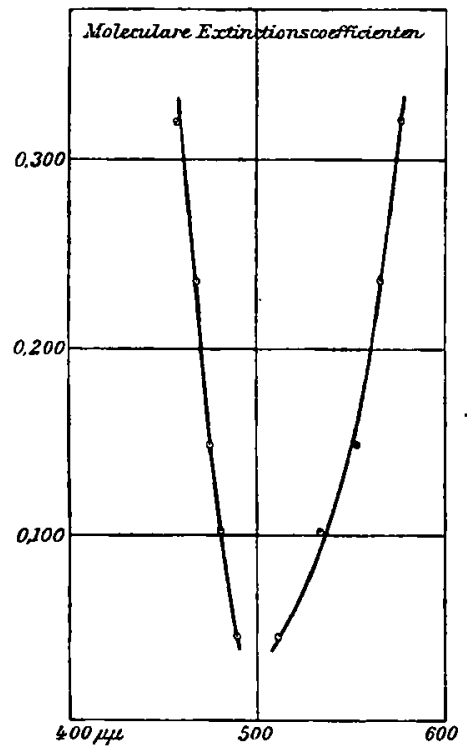

Fig. 11.
Letztere Absorption ist offenbar die Ursache davon, daß die Abweichungen rom Beerschen Gesetz bei $\mathrm{NiCl}_{2}$ und $\mathrm{NiBr}_{2}$ bedeutend geringer sind, als bei $\mathrm{CuCl}_{2}$ und $\mathrm{CuBr}_{2}$.

Die Farbe von $\mathrm{NiSO}_{4}$ und $\mathrm{Ni}\left(\mathrm{NO}_{3}\right)_{2}$ zeigt sich unabhängig von der Temperatur, dagegen gilt für $\mathrm{NiCl}_{2}$ und $\mathrm{NiBr}_{2}$ wieder der Satz, daB Erhöhung der Temperatur auf die Lichtabsorption denselben Einfluß hat wie $\nabla$ ergrößerung, Erniedrigung der Temperatur denselben EinfluB hat wie Verringerung der Konzentration.

§ 12. Zusammenstellung der Resultate. 1. Das $\mathrm{Cu}$ übt im sichtbaren Gebiet in den verdünnten Lösungen aller unter- 
suchten Kupfersalze, sowie in den konzentrierten Lösungen von $\mathrm{CuSO}_{4}, \mathrm{CuCl}_{2}, \mathrm{CuBr}_{2}$ die gleiche Absorption aus, und $\mathrm{zwar}$ absorbiert das $\mathrm{Cu}$ das rote Ende des sichtbaren Spektrums. In den verdünnten Lösungen tritt auBer dieser durch $\mathrm{Cu}$ bewirkten Absorption keine weitere Absorption auf. Auch in der konzentrierten Lösung von $\mathrm{CuSO}_{4}$ tritt zu der durch das $\mathrm{Cu}$ bewirkten Absorption keine weitere Absorption hinzu. Dagegen tritt in den konzentrierten Lösungen von $\mathrm{CuCl}_{2}$ und $\mathrm{CuBr}_{2}$ zu der durch das $\mathrm{Cu}$ bewirkten Absorption des Rot noch eine solche des Blau und Violett hinzu, welche wahr. scheinlich durch $\mathrm{Cl}_{2}$ bez. $\mathrm{Br}_{2}$ bewirkt wird. In den konzentrierten Lösungen von Kupfernitrat und Kupferacetat tritt zu der $\mathrm{Cu}$-Absorption der verdünnten Lösung noch eine weitere Absorption des Rot hinzu.

2. Bei zunebmender Verdünnung nimmt der molekulare Extinktionskoeffizient bei den untersuchten Kupfersalzen einen bestimmten Grenzwert $A_{0}$ an; ist dieser erreicht, so ist weitere Verdünnung auf die molekulare Lichtabsorption ohne Einflub. Der Grenzwert $A_{0}$ ist in Übereinstimmung mit der KnoblauchOstwaldschen Theorie im ganzen sichtbaren Gebiet für alle untersuchten Kupfersalze der gleiche; daher ist die molekulare Extinktionskurve der verdünnten Kupfersalzlösungen für die Absorption des $\mathrm{Cu}$ charakteristisch.

3. Auch bei den Nickelsalzen ist das Beersche Gesetz im allgemeinen nicht erfüllt; die Abweichungen sind hier von derselben Art, wie bei den entsprechenden Kupfersalzen; sie sind jedoch mit Ausnahme von $\mathrm{NiCl}_{2}$ und $\mathrm{NiBr}_{2}$ äußerst gering. Auch die Nickelsalze weisen bei genügender Verdünnung den gleichen grünen Farbenton auf, obwohl die konzentrierten Lösungen zum Teil sehr verschiedene Farbe besitzen; dieser Farbenton ist demnach für $\mathrm{Ni}$ charakteristisch, und zwar absorbiert $\mathrm{Ni}$ das rote und das blaue Ende des sichtbaren Spektrums.

4. Diejenigen Salzlösungen, bei denen das Beersche Gesetz im ganzen sichtbaren Gebiet erfüllt ist, zeigen keine Abhängigkeit ihrer Farbe von der Temperatur, während auf die Farbe der anderen untersuchten Salzlösungen Temperaturerhöhung denselben Einfluß hat wie VergröBerung, Abkühlung denselben EinfluB wie Verringerung der Konzentration. 
5. Der wahrscheinliche Grund für die Nichterfüllung des Beerschen Gesetzes ist in der Dissoziation zu sehen. Hierfür spricht die Tatsache, daB die molekularen Extinktionskoeffizienten bei zunehmender Verdünnung einen bestimmten Grenzwert erreichen, und daB dieser Grenzwert in Übereinstimmung mit der Knoblauch-Ostwaldschen Theorie für alle untersuchten Kupfersalze der gleiche ist. Auch die schon erwähnte Abhängigkeit der Lichtabsorption von der Temperatur spricht hierfür, insofern die Änderung der Temperatur einer Lösung eine Änderung des Dissoziationsgrades zur Folge hat.

Vorliegende Arbeit wurde im physikalischen Institut der Universität Berlin angefertigt. Es sei mir gestattet, dem Leiter desselben, meinem hochverehrten Lehrer Hrn. Geh.-Rat Prof. Dr. Warburg für die Anregung zu dieser Arbeit, sowie das freundliche Interesse an derselben meinen ehrerbietigsten Dank auszusprechen.

Auch Hrn. Privatdozenten Dr. F. F. Martens bin ich für die wertvollen Ratschläge, mit denen er die Arbeit zu fördern immer bemüht war, zu stetem Dank verpflichtet.

Aachen, 30. Juni 1903.

(Eingegangen 18. Juli 1903.) 